\title{
Co-receptor usage prediction at quasispecies level using ultra-deep pyrosequencing on both circulating and proviral hiv in patients candidates to CCR5 antagonist treatment
}

\author{
Isabella Abbate*, Gabriella Rozera, Chiara Tommasi, Alessandro Bruselles, Barbara Bartolini, Emanuele Nicastri, \\ Pasquale Narciso, Maria R Capobianchi
}

From $16^{\text {th }}$ International Symposium on HIV and Emerging Infectious Diseases Marseille, France. 24-26 March 2010

\section{Background}

Ultra-deep Pyrosequencing (UDPS) offers the opportunity to analyze the co-receptor usage of each variant present in a viral quasispecies. Aim of the study was to assess co-receptor usage by UDPS, in comparison with a reference phenotypic test, in patients candidates to CCR5 antagonists treatment, in both circulating and proviral HIV-1.

\section{Methods}

Seventeen patients were enrolled. Trofile phenotypic test was performed by standard in 9 and by enhanced procedures in 8 patients. UDPS was carried out on both plasma virus RNA and on PBMC proviral DNA, amplifying env V3 loop region. Genotypic prediction of coreceptor usage was obtained by Position Specific Score Matrix analysis. Comparison between phenotypic and genotypic assays was performed considering a threshold of $5 \%$ and $0.3 \%$ for $\mathrm{X} 4$ detection (standard and enhanced Trofile sensitivity).

\section{Results}

After editing, a total of 130,999 V3 sequences were considered, with a mean coverage per site of 5,955. Concordance between phenotypic and UDPS results was $0.60 \pm 0.24$ (Cohen K index $\pm \mathrm{SE}$ ). Discordant results were observed in one case with standard and in 2 cases with enhanced trofile test. Only one patient, eligible to CCR5 antagonist treatment based on Trofile results, showed $\mathrm{X} 4$ variants in the circulation by UDPS at a frequency higher than that of Trofile sensitivity. Quasispecies archived in PBMC tended to be more heterogeneous than that found in circulating virus. All patients, with only one exception, harboured in proviral DNA X4 variants at variable frequency (from $0.07 \%$ to $53 \%)$.

\section{Discussion}

UDPS allows a detailed characterization of HIV V3 quasispecies in both circulating and archived sequences. Almost concordant results were obtained by UDPS and Trofile for prediction of co-receptor usage of plasma virions. In proviral DNA, X4 variants were commonly observed at variable frequencies. The importance of archived X4 variants in influencing clinical response to CCR5 antagonists is a crucial point to be addressed.

Published: 11 May 2010

* Correspondence: abbate@inmi.it

INMI L.Spallanzani, Rome, Italy 